\title{
Systematic investigation of biomass and lipid productivity by microalgae in photobioreactors for biodiesel application
}

\author{
J. Pruvost ${ }^{\mathrm{a}, *}$, G. Van Vooren ${ }^{\mathrm{a}}$, B. Le Gouic ${ }^{\mathrm{a}}$, A. Couzinet-Mossion ${ }^{\mathrm{b}}$, J. Legrand ${ }^{\mathrm{a}}$ \\ a Université de Nantes, CNRS, GEPEA UMR-CNRS 6144, bd de l'Université, CRTT-BP 406, 44602 Saint-Nazaire Cedex, France \\ ${ }^{\mathrm{b}}$ Université de Nantes, Groupe Mer, Molécules, Santé, EA 2160, Faculté de Pharmacie, Pôle Mer et Littoral, 1 rue G. Veil, BP 53508, F-44035 Nantes Cedex 1, France
}

Keywords:

Microalgae

Biodiesel

TAG

Photobioreactor

Lipid

\begin{abstract}
A B S T R A C T
We describe a methodology to investigate the potential of given microalgae species for biodiesel production by characterizing their productivity in terms of both biomass and lipids. A multi-step approach was used: determination of biological needs for macronutrients (nitrate, phosphate and sulphate), determination of maximum biomass productivity (the "light-limited" regime), scaling-up of biomass production in photobioreactors, including a theoretical framework to predict corresponding productivities, and investigation of how nitrate starvation protocol affects cell biochemical composition and triggers triacylglycerol (TAG) accumulation. The methodology was applied to two freshwater strains, Chlorella vulgaris and Neochloris oleoabundans, and one seawater diatom strain, Cylindrotheca closterium. The highest total lipid content was achieved with $N$. oleoabundans (25-37\% of DW), while the highest TAG content was found in C. vulgaris (11-14\% of DW). These two species showed similar TAG productivities.
\end{abstract}

\section{Introduction}

Microalgae display high areal productivity, and some of them are able to accumulate significant amounts of lipids. They are therefore seen as promising candidates for the industrial production of biodiesel. To expand this novel feedstock, research and development is needed in several domains, from the selection of suitable strains to the optimization of the different steps required for mass scale operation (biomass production, harvesting, lipid extraction).

Quantity, quality and productivity of lipid are obviously of primary relevance. They depend not only on the strains, but also on culture conditions; for example, it is well known that nitrate starvation can trigger lipid accumulation, especially triacylglycerols (TAGs) suitable for biodiesel production (Chisti, 2007; Hu et al., 2008; Rodolfi et al., 2009). Finding the best species implies screening a broad biodiversity of microalgae and characterizing their productivity. This task is less straightforward than it may seem. Optimization involves controlling a range of parameters affecting productivity. For photosynthetic organisms such as microalgae, this requires especially controlling the light supply, the main variable governing growth. This was recently studied in Chlamydomonas reinhardtii in the general case of maximum biomass productivity achievement (Takache et al., 2010) and also in the specific case of hydrogen production (Degrenne et al., 2010;
Fouchard et al., 2008). For both biomass and hydrogen, the welldefined conditions of a lab-scale photobioreactor (PBR) enabled the influence of culture parameters on productivities to be successfully studied.

We conducted analogous experiments in lab-scale PBRs to investigate lipid production by Neochloris oleoabundans, a species known for its ability to accumulate lipids, and especially TAG (Tornabene et al., 1983). Lipid productivities were quantified in conditions maximizing biomass productivity, and also under nitrogen starvation, which triggers lipid accumulation. Similar productivities were observed for TAG irrespective of the protocol tested. Although nitrate starvation was necessary to induce TAG accumulation ( $18 \%$ of dry weight), TAG productivity was sustained when operating at maximum biomass productivity, because of natural TAG content in $N$. oleoabundans as obtained in continuous mode in light-limited condition ( $3 \%$ of dry weight). These results emphasize the difficulty quantifying the utility of a strain for lipid production because of the close dependence on culture conditions and the production strategies applied.

This work is extended here by applying the procedure to Chlorella vulgaris, a freshwater species also known for lipid accumulation (Rodolfi et al., 2009; Scragg et al., 2002), and because its mass production at industrial scale has already been demonstrated (Doucha and Livansky, 2006; Richmond, 2004). Nitrogen starvation effects were characterized by investigating changes in the biochemical composition of $N$. oleoabundans and $C$. vulgaris strains. $N$. oleoabundans production was finally scaled up in a $0.130 \mathrm{~m}^{3}$ airlift PBR. Results are compared with those of a model representing 
light-limited growth in a PBR. This model applied to N. oleoabundans was validated in PBRs of different geometries, with volumes from $10^{-3}$ to $0.130 \mathrm{~m}^{3}$, illustrating the usefulness of a theoretical approach in the scaling-up of such systems.

\section{Methods}

\subsection{Strains}

Three strains were investigated: two freshwater green algae, $N$. oleoabundans (strain 1185) and C. vulgaris (strain CCAP 211/19) (Chlorophyceae), and C. closterium, a marine diatom. The first was obtained from the culture collection of the University of Austin (Texas, USA). C. vulgaris and C. closterium were collected from the culture collection of IFREMER-Nantes (Nantes, France). Growth media for the two freshwater strains $N$. oleoabundans and C. vulgaris were based on Bold Basal Medium (BBM). Growth medium for $C$. closterium was based on natural seawater from the coastal area of Saint-Nazaire in France, filter-sterilized through a $0.22 \mu \mathrm{m}$ pore size filter, enriched with Conway medium ( $3 \mathrm{~mL} / \mathrm{L}$ of seawater) and a $40 \mathrm{~g} / \mathrm{L}$ stock solution of $\mathrm{Na}_{2} \mathrm{SiO}_{3}, 5 \mathrm{H}_{2} \mathrm{O}$ (1 mL/L of seawater). Each medium was adjusted to avoid mineral limitation as described below. Exact composition of BBM and Conway medium are given in Pruvost et al. (2009) and Loubiere et al. (2009), respectively.

\subsection{Design of the growth media}

Only photoautotrophic growth was investigated here. To obtain "light-limited" conditions, the growth medium must contain all the necessary nutrients (macro and micro) in sufficient quantities, and must thus be adjusted according to the biomass concentration planned. This has already been discussed for N. oleoabundans (Pruvost et al., 2009). The same procedure was applied here (only the main aspects are given. Refer to the earlier work for full details).

As a first estimate of macronutrient requirements, the elemental composition of each strain was determined. The biomass content of elements $\mathrm{C}, \mathrm{H}, \mathrm{O}, \mathrm{N}, \mathrm{S}, \mathrm{P}$ can then be used to define an overall stoichiometry translating elements into nitrate (or ammonium, depending on the nitrogen source chosen), phosphate and sulphate concentrations (see Roels (1983)) for the general method). Macronutrient requirement was then checked by measuring mineral consumption by anionic chromatography during batch culture (again see Pruvost et al. (2009) for an example).

For micronutrients, elementary analysis is also possible but difficult due to the very low concentrations (Cogne et al., 2003). Standard media composition for micronutrients was thus generally adopted and tested for possible limitations in a second step. After macronutrient formulation is chosen, a continuous culture is conducted in a PBR and the quantity of micronutrients is then doubled. Any increase in biomass concentration (over at least six residence times to obtain biomass and medium steady-state) indicates a prior limitation of one or more micronutrients. This approach was used here.

One advantage of precisely controlling macronutrients formulation is the possibility of planning nitrate starvation, without sulphate or phosphate limitations, to increase lipid production. Biomass from a PBR operating in continuous mode with no limitations but with most of the nitrates consumed may be used to inoculate a second PBR operating in batch mode where nitrate consumption during initial growth initiates starvation promptly (progressive starvation protocol described in Pruvost et al. (2009)). This experiment can be repeated, as the PBR operating in continuous mode can supply the same biomass and media every day. However, because the time taken to obtain nitrate starvation depends on the rate of consumption of each species, we elected to centrifuge and then re-suspend cells in a nitrate-free medium under light $\left(q_{0}=220 \mu\right.$ mole $\left.\mathrm{m}^{-2} \mathrm{~s}^{-1}\right)$. Nitrate depletion was thus applied from the start of each experiment (sudden deprivation protocol also described in Pruvost et al. (2009)). Cells were harvested from a PBR operating in continuous mode to ensure the same initial physiological state.

\subsection{Description of photobioreactors}

All the small-scale experiments were conducted in flat-panel airlift photobioreactors (PBR1) of a volume $V_{\mathrm{r}}=10^{-3} \mathrm{~m}^{3}$ and depth of culture $L_{z}=0.03 \mathrm{~m}$, giving a specific illuminated area $a_{\text {light }}=S / V_{\mathrm{r}}$ of $33.3 \mathrm{~m}^{-1}$ (Fig. 1a). Four PBR were used in parallel for faster screening of operating conditions. Each one was controlled for temperature and $\mathrm{pH}$ (by automatic injection of pure $\mathrm{CO}_{2}$ ) and equipped for continuous production. A more complete description is given in Pruvost et al. (2009).

A second type of PBR was especially designed for scaling up production in controlled conditions (Fig. 1b). This system (PBR2) had a volume $V_{\mathrm{r}}=0.13 \mathrm{~m}^{3}$ with a greater depth of culture $L_{z}=0.055 \mathrm{~m}$, giving a specific illuminated area $a_{\text {light }}=S / V_{\mathrm{r}}$ of $18.2 \mathrm{~m}^{-1}$. PBR2 was made of stainless steel (quality $316 \mathrm{~L}$ ), except for the optical surface (front side), which was made of transparent polycarbonate. Both PBR1 and PBR2 were pneumatically agitated. PBR2 was illuminated on two sides using 20 fluorescent tubes (Sylvania luxline plus F58W/860). Various values of incident photon flux density (PFD) could be applied simply switching on appropriate number of fluorescent tubes. All the PBR used in this study (flat panel type PBR) present one-dimensional light attenuation along the depth of culture $z$, perpendicular to the optical flat surface (see Pottier et al. (2005) for details). As described below, this option was chosen to facilitate the control and modelling of radiative transfer conditions inside the culture (see next section for details).

PFD was measured using a plane cosine quantum sensor (LICOR LI-190-SA, Lincoln, NE), which quantifies photosynthetically active radiation (PAR) in the $400-700 \mathrm{~nm}$ waveband received in a $2 \pi$ solid angle. PFD was measured on the rear side of the optical surface to take into account attenuation of light travelling through this surface (average of several measuring locations). For all the experiments in PBRs, a controlled temperature of $25^{\circ} \mathrm{C}$ and $\mathrm{pH}$ of 7.5 were set (sensor Mettler Toledo SG 3253) by air blowing and $\mathrm{CO}_{2}$ injection respectively. All the PBR could be run in batch or continuous (chemostat) production mode. In the latter case, the input liquid flow rate was controlled using a dosing pump for PBR1 (Stepdos ${ }^{\circledR}$ pump 08/RC, KNF Neuberger) and a pump combined with a mass flow-meter (Bronkhorst) for PBR2. For all the PBRs, the culture was harvested by overflowing, keeping the volume of the reactor constant.

\subsection{Analysis}

Methods for measuring dry weight, elemental composition, total lipids and nutrients (nitrate, sulphate and phosphate) have already been described in Pruvost et al. (2009). Measuring fluorescence to estimate photosynthetic activity for different irradiances is described in Takache et al. (2010). Here we added the following analysis:

- Pigment content: pigment content was determined using a spectrophotometric method. A volume $V_{1}$ of culture was centrifuged for $5 \mathrm{mn}$ at $13,400 \mathrm{rpm}$ in a $2 \mathrm{~mL}$ tube, the supernatant was eliminated and the pellet was extracted with $1.5 \mathrm{~mL}$ methanol (99.9\%) for $30 \mathrm{~min}$ in the dark at $45^{\circ} \mathrm{C}$. Samples were centrifuged $(13,400 \mathrm{rpm}, 5 \mathrm{~min})$ before measurements. Absorption spectra were collected in the range $400-750 \mathrm{~nm}$ (spectropho- 


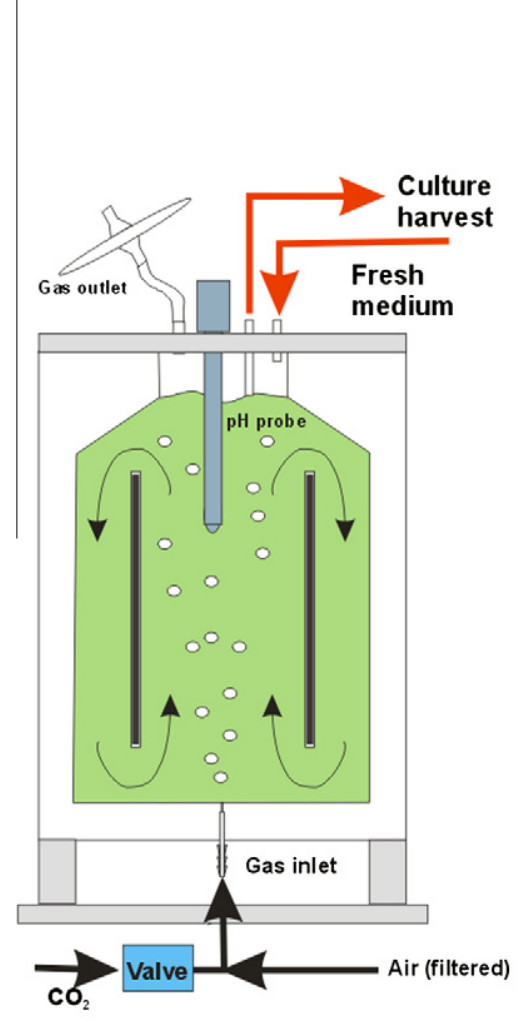

b

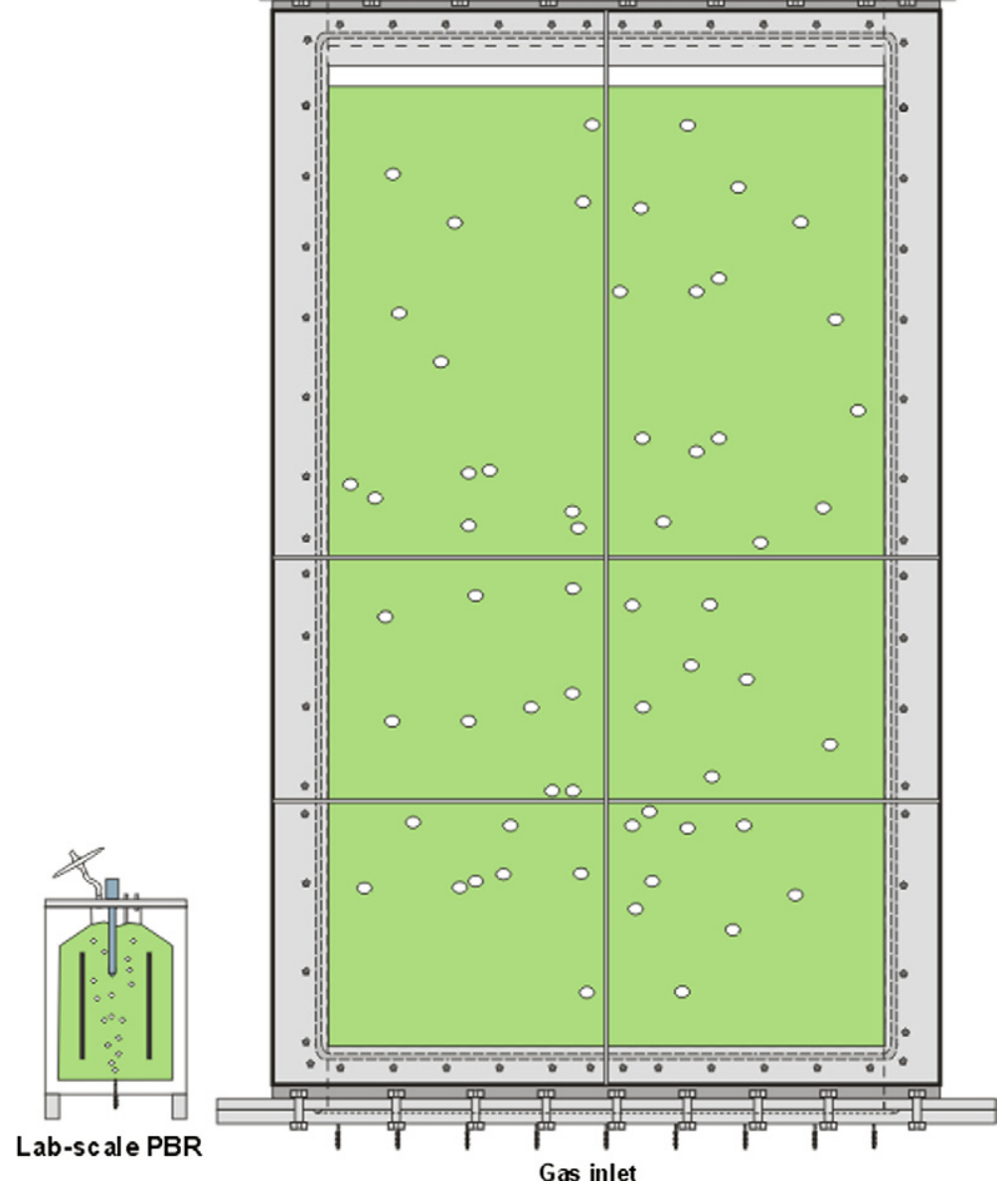

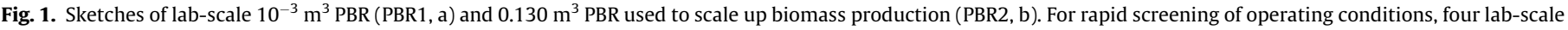
PBR were used in parallel. Both PBRs are airlift flat-panel systems allowing both batch and continuous production.

tometer Jenway 6500) in a cell with a path length of $1 \mathrm{~cm}$. Chlorophyll-a (Chl-a), chlorophyll-b (Chl-b) and photoprotective carotenoid (PPC) concentrations were determined according to the equations of Ritchie (2006) for chlorophylls and Strickland and Parsons (1968) for carotenoids:

$\left[\right.$ Chl-a] $\mu \mathrm{g} / \mathrm{mL}=-8.0962 \times A_{652}+16.5169 \times A_{665} ;$

$\left[\right.$ Chl-b] $\mu \mathrm{g} / \mathrm{mL}=27.4405 \times A_{652}-12.1688 \times A_{665}$;

[Carotenoids] $\mu \mathrm{g} / \mathrm{mL}=4 \times A_{480}$.

Absorbencies at 480, 652 and $665 \mathrm{~nm}$ were corrected for turbidity by subtracting absorbencies at $750 \mathrm{~nm}$. Results given are an average of three measurements. $V_{1}$ was chosen to obtain absorbances between 0.1 and 0.7 .

- Protein content: cells were first subjected to an alkaline lyses followed by an acid neutralization and protein concentration was finally determined by the Lowry method (Lowry et al., 1951). A volume $V_{1}$ of culture was centrifuged for $10 \mathrm{~min}$. at $13,400 \mathrm{rpm}$ in a $2 \mathrm{~mL}$ tube, the supernatant was discarded and the pellet was suspended in a volume $V_{2}$ of deionised water. One millilitre of this solution was mixed with $1 \mathrm{~mL}$ of $2 \mathrm{~N} \mathrm{NaOH}$ in a $15 \mathrm{~mL}$ plastic tube and heated in a water bath at $95^{\circ} \mathrm{C}$ for $6 \mathrm{~min}$. and left to then cool. One millilitre of $1.6 \mathrm{~N} \mathrm{HCl}$ was added for neutralization. This solution was used for protein measurement by the Lowry method. BSA was used as protein standard in the range $0.2-0.4 \mathrm{~g} / \mathrm{L}$. Dilution or concentration factor $V_{2} / V_{1}$ was chosen inside this BSA range.

- Total sugar content: total sugar content was determined by the phenol-sulphuric acid method of Dubois et al. (1956). A volume $V_{1}$ of culture was centrifuged for $10 \mathrm{~min}$. at $13,400 \mathrm{rpm}$ in a
$2 \mathrm{~mL}$ tube, the supernatant was discarded and the pellet was suspended in a volume $V_{2}$ of deionised water. $0.5 \mathrm{~mL}$ of this solution was added to $0.5 \mathrm{~mL}$ of a $50 \mathrm{~g} / \mathrm{L}$ phenol solution in a $15 \mathrm{~mL}$ polypropylene tube. No mixing was done and careful attention was paid to avoid any drops on the walls. $2.5 \mathrm{~mL}$ of 95-97\% $\mathrm{H}_{2} \mathrm{SO}_{4}$ was added rapidly directly on the liquid surface. After $10 \mathrm{~min}$. of incubation the tube was vortexed vigorously for 10 ', let at room temperature and then placed in a water bath at $35^{\circ} \mathrm{C}$ for $30 \mathrm{~min}$. Absorbance was measured at $483 \mathrm{~nm}$. Glucose was used as a standard in the range $0.02-0.08 \mathrm{~g} / \mathrm{L}$ and dilution factor $V_{2} / V_{1}$ was chosen accordingly.

- TAGs: total lipids including chlorophyll and pigments were extracted by the Folch method and weighed on a $0.1 \mathrm{mg}$ laboratory scale. Initial culture volume was chosen to obtain a total weight of lipid including pigments in the range $7-10 \mathrm{mg}$. Three replicates were done (estimated precision was $\pm 5 \%$ ). The ratio of total lipids was obtained relative to dry biomass (three replicates in the range $7-10 \mathrm{mg}$ ). Neutral lipids and especially TAG were separated from the other lipids by column chromatography on silica gel (GracePur, $500 \mathrm{mg}$, Alltech) using $\mathrm{CHCl}_{3} / \mathrm{MeOH}$ 98/2 v/v\% (neutral lipids), $\mathrm{CHCl}_{3} / \mathrm{MeOH}$ 5:1 v/v\% (glycolipids) and $\mathrm{MeOH}$ (phospholipids). The TAG fraction was collected separately and weighed, and its purity was checked using thinlayer chromatography on $20 \mathrm{~cm} \times 20 \mathrm{~cm} \times 0.25 \mathrm{~mm}$ silica gel plates (polygram sil G, Alltech, France). The plates were developed in hexane-diethyl ether-acetic acid (60:15:0.75, v/v/v) and stained using iodine vapour. For TAG, precision was estimated at $\pm 10 \%$. 


\subsection{Theoretical considerations}

\subsubsection{Light-limited growth model}

The biomass concentration $C_{x}$ can be obtained by a standard mass balance on a continuous PBR assuming perfectly mixed conditions (Cornet et al., 2003; Pruvost et al., 2008):

$\frac{d C_{X}}{d t}=\left\langle r_{X}\right\rangle-\frac{C_{X}}{\tau}$

with $C_{x}$ the biomass concentration, $\left\langle r_{x}\right\rangle$ the mean biomass volumetric growth rate in the PBR, and $\tau$ the residence time for the PBR resulting from the liquid flow rate of the feed (fresh medium) (with $\tau=1 / D$, where $D$ is the dilution rate).

Solving Eq. (1) implies determining the mean volumetric growth rate $\left\langle r_{x}\right\rangle$. In light-limited conditions, the growth rate is linked only to the light received. However, it is well known that cells absorb and scatter light, leading to a heterogeneous light distribution inside the PBR culture volume. This results in a local value of the volumetric growth rate $r_{x}$ that has to be averaged over piration term $\left(r_{x, r}\right)$ dependant on the biomass concentration. This is the same as introducing a constant specific respiration rate $\mu_{s}$, giving:

$r_{x, r}=-\mu_{s} C_{X}$

Though at first sight as over-simple, this formulation was shown to be sufficient in the case investigated here $(N$. oleoabundans). This can be explained by the low respiration activity observed in light for this strain (as shown later). Ultimately, the contribution of respiration to the overall growth rate remains low.

To extend the model, the irradiance-field in the PBR had to be given (Eq. (4)). The irradiance-field is a function of PBR geometry, light source and cell optical properties. All the PBRs investigated in this study met to the "one-dimensional" hypothesis, where light attenuation occurs mainly along a single direction perpendicular to the illuminated surface (the depth of culture). The two-flux model was found to give accurate results in this case (Pottier et al., 2005). The irradiance profile $G(z)$ along the depth of culture $z$ is then given by the following equation:

$\frac{G(z)}{q_{0}}=2 \frac{[(\chi(1+\alpha) \exp (-\delta L)-(1-\alpha) \exp (-\delta L) \exp (\delta z)+(1+\alpha) \exp (\delta L)-\chi(1-\alpha) \exp (\delta L)] \exp (\delta z)}{(1+\alpha)^{2} \exp (\delta L)-(1-\alpha)^{2} \exp (-\delta L)-\chi\left(1-\alpha^{2}\right) \exp (\delta L)+\chi\left(1-\alpha^{2}\right) \exp (-\delta L)}$

the reactor volume to obtain the mean volumetric growth rate $\left\langle r_{x}\right\rangle$. For a PBR with one-dimensional light attenuation, this consists in a simple integration along the depth of culture $z$ :

$\left\langle r_{x}\right\rangle=\frac{1}{L} \int_{0}^{L} r_{x} \cdot d z$

where $L$ is the photobioreactor depth.

Formulation of the volumetric growth rate $r_{x}$ is obviously a key step in the modelling. Numerous kinetic models exist for describing the growth of photosynthetic microorganisms (Aiba, 1982; Muller-Feuga, 1998). For photosynthetic eukaryotic cells such as microalgae, growth is the result of the biomass increase caused by photosynthesis in chloroplasts (anabolism) and its partial degradation by respiration in mitochondria (catabolism). The total growth rate can thus be expressed as the sum of two terms (Fouchard et al., 2009):

$r_{x}=r_{x, p}+r_{x, s}$

with $r_{x, p}$ and $r_{x, s}$ related respectively to the photosynthetic growth and the respiration process.

Cornet and Dussap (2009) provide an interesting formulation by linking the photosynthetic growth rate to the local radiant light power density absorbed $\mathscr{A}$ and thus the local value of irradiance $G$ inside the PBR, according to:

$r_{x, p}=\rho \bar{\phi} \mathscr{A}=\rho_{M} \frac{K}{K+G} \bar{\phi} E a G C_{X}$

where $G$ the irradiance, $\rho_{M}$ the maximum energetic yield for photon conversion, $\bar{\phi}$ the mass quantum yield for the Z-scheme of photosynthesis, $K$ the half saturation constant for photosynthesis and $E a$ the mass absorption coefficient.

This formulation was applied successfully to the cyanobacteria Arthrospira platensis. As a prokaryotic cell, with therefore a common electron carrier chain for photosynthesis and respiration, $A$. platensis displays no respiration in light (Gonzalez de la Vara and Gomez-Lojero, 1986). This simplify the kinetic formulation by setting $r_{x, r}=0$, which may not be assumed for microalgae. Thus here we opted to adapt the formulation by introducing a catabolism res- where $q_{0}$ is the hemispherical incident light flux (same as PFD) and $\chi$ is the reflection coefficient of the wall defining the depth of culture. This wall was made of stainless steel for all the PBR used in the present study (for cooling). The reflection coefficient was thus equal to 0.51 (Takache et al., 2010).

In Eq. (6), $\alpha$ (linear scattering modulus) and $\delta$ (two flux extinction coefficient) are given by:

$$
\alpha=\sqrt{\frac{E a}{E a+2 b E s}} \quad \delta=C_{x} \sqrt{E a(E a+2 b E s)}
$$

where $E a$ and $E s$ are the absorption and scattering mass coefficients respectively, and $b$ is the backscattered fraction.These three values defined the optical properties used in the two-flux model. They were determined here for $N$. oleoabundans (see below).

\subsubsection{Determination of model parameters for $N$. oleoabundans}

The light-limited growth model described above requires determining several variables. Radiative properties are deduced from pigment content measurement and image analysis provides cell shape and size. The experimental procedure is the same as described in Pottier et al. (2005). N. oleoabundans cells were considered as Tchebytchev particles T2(0.043) of order two, with a deformation parameter $\varepsilon=0.043$ (Mishchenko et al., 1998). A size distribution of log-normal type with an equivalent radius of $3.2 \mu \mathrm{m}$ and standard deviation $\sigma=1.16$ was retained. Based on this analysis, a predictive approach, applying the generalized LorenzMie theory with the anomalous diffraction approximation (Cornet, 2007; Van de Hulst, 1981), was then used to calculate corresponding radiative properties (i.e. the mass absorption coefficient $E a$, the scattering coefficients $E s$ and the backscattered fraction $b$ ). These values are a function of the pigment contents. For $N$. oleoabundans (classical growth conditions) we found a mass content of $4 \%, 1 \%$ and $1.6 \%$ of chlorophyll-a, chlorophyll-b and carotenoids respectively. Corresponding radiative properties were $E a=360 \mathrm{~m}^{2} \mathrm{~kg}^{-1}$, Es $=2380 \mathrm{~m}^{2} \mathrm{~kg}^{-1}$ and $b=0.003$. The linear scattering modulus $\alpha$ was thus equal to 0.98 .

The determination of kinetic parameters for the photosynthetic growth model requires various measurements and methods. A full 
description of each lies outside of the scope of this work, but detailed procedures may be found elsewhere (Cornet and Dussap, 2009; Takache et al., 2010). Following Cornet and Dussap (2009), the maximum energy yield for photon dissipation in antennae was set to $\rho_{M}=0.8$, as a moderately species-independent value. The mean spatial quantum yield for the Z-scheme of photosynthesis was calculated from the elementary composition $\left(\mathrm{CH}_{1.715} \mathrm{O}_{0.427} \mathrm{~N}_{0.148} \mathrm{~S}_{0.014} \mathrm{P}_{0.012}\right)$ of $N$. oleoabundans (Pruvost et al., 2009), giving the value $\bar{\phi}=1.83 \times 10^{-9} \mathrm{~kg} \mu$ mole $_{\mathrm{h} v}{ }^{-1}$. The two remaining parameters are the half saturation constant $K$ and the specific respiration rate $\mu_{s}$. The first was estimated as in Takache et al. (2010) for Chlamydomonas reinhardtii by using the saturation pulse method to measure the maximum PSII yield (maximum yield $=\mathrm{Fv} / \mathrm{Fm}$ ) as a function of irradiance (Schreiber et al., 1998) using a WaterPam chlorophyll fluorometer (Walz, Gemany). The second was estimated by biomass loss during a dark period. Both values were finally adjusted by global regression on experimental data (permitting a maximum variation of $30 \%$ on parameters), leading finally to $K=90 \mu \mathrm{mol}_{\mathrm{hv}} \mathrm{m}^{-2} \mathrm{~s}^{-1}$ and $\mu_{\mathrm{s}}=5 \times 10^{-3} \mathrm{~h}^{-1}$ for N. oleoabundans.

\subsubsection{Biomass productivity prediction}

Biomass concentration in light-limited conditions was obtained by solving the mass balance equation (Eq. (1)) (Matlab software) using the kinetic growth (Eqs. (4) and (5)) and radiative models. For continuous mode, at steady-state $\left(d C_{x} / d t=0\right)$, this gives the volumetric biomass productivity $\left\langle r_{x}\right\rangle$ directly. For a given species (characterized by its optical properties and kinetic growth parameters), this value will be a function of operating parameters, such as depth of culture, dilution rate (or residence time $\tau$ ) or incident PFD. As will be demonstrated here, this makes this tool of primary relevance in process optimization and prediction of PBR productivity evolution as a function of those key parameters.

More directly (without numerical resolution), the maximum biomass productivity $\left\langle r_{x}\right\rangle_{\max }$ can also be obtained. This was recently proposed by Cornet and Dussap (2009), who observed that maximum productivity was obtained for the particular case of full light absorption, but with no dark zone (the so-called luminostat regime). This allows an analytical solution for maximum biomass volumetric productivity to be calculated, so for the flat-panel PBR investigated here:

$$
\left\langle r_{x}\right\rangle_{\max }=\rho_{\mathrm{M}} \bar{\phi} \frac{2 \alpha}{1+\alpha} a_{\text {light }} K \ln \left[1+\frac{q_{\cap}}{K}\right]
$$

or in terms of maximum areal biomass productivity:

$$
\left\langle S_{x}\right\rangle_{\max }=\rho_{M} \bar{\phi} \frac{2 \alpha}{1+\alpha} K \ln \left[1+\frac{q_{\cap}}{K}\right]
$$

All the parameters required are presented above. This equation was applied successfully (Takache et al., 2010) on the microalga Chlamydomonas reinhardtii in two different PBR geometries (toric and cylindrical PBR). It was tested here with $N$. oleoabundans cultivated in different PBRs (all of the flat panel type).

\section{Results and discussion}

3.1. Characterization of the maximum biomass productivity in lightlimited conditions for the three different species

As a preliminary step, light-limited growth was investigated for C. vulgaris and C. closterium and compared with results obtained with N. oleoabundans (Pruvost et al., 2009). Mineral limitation was avoided by following the medium design procedure described in Section 2.2 and by checking mineral concentration during cultivation (all concentrations were found to exceed $100 \mathrm{mg} / \mathrm{L}$ for nitrate, and $30 \mathrm{mg} / \mathrm{L}$ for phosphate and sulphate). It results in a productivity that is a function of only the light supply, as set by the PFD received on the PBR surface and by the light transfer conditions inside the reactor volume ("light-limited" growth conditions). As a result, for a given PFD, the dilution rate affects biomass concentration and thus light transfer conditions. If the biomass concentration is too low, some of the light is transmitted through the culture (low absorption, favouring the "kinetic" regime). Conversely, if the biomass is too high, a dark zone appears in the depth of culture (favouring the light limitation regime). Both situations result in a loss of biomass productivity. In the first case, the light is not fully absorbed, while in the second case, the dark zone, where respiration is predominant, has a negative influence

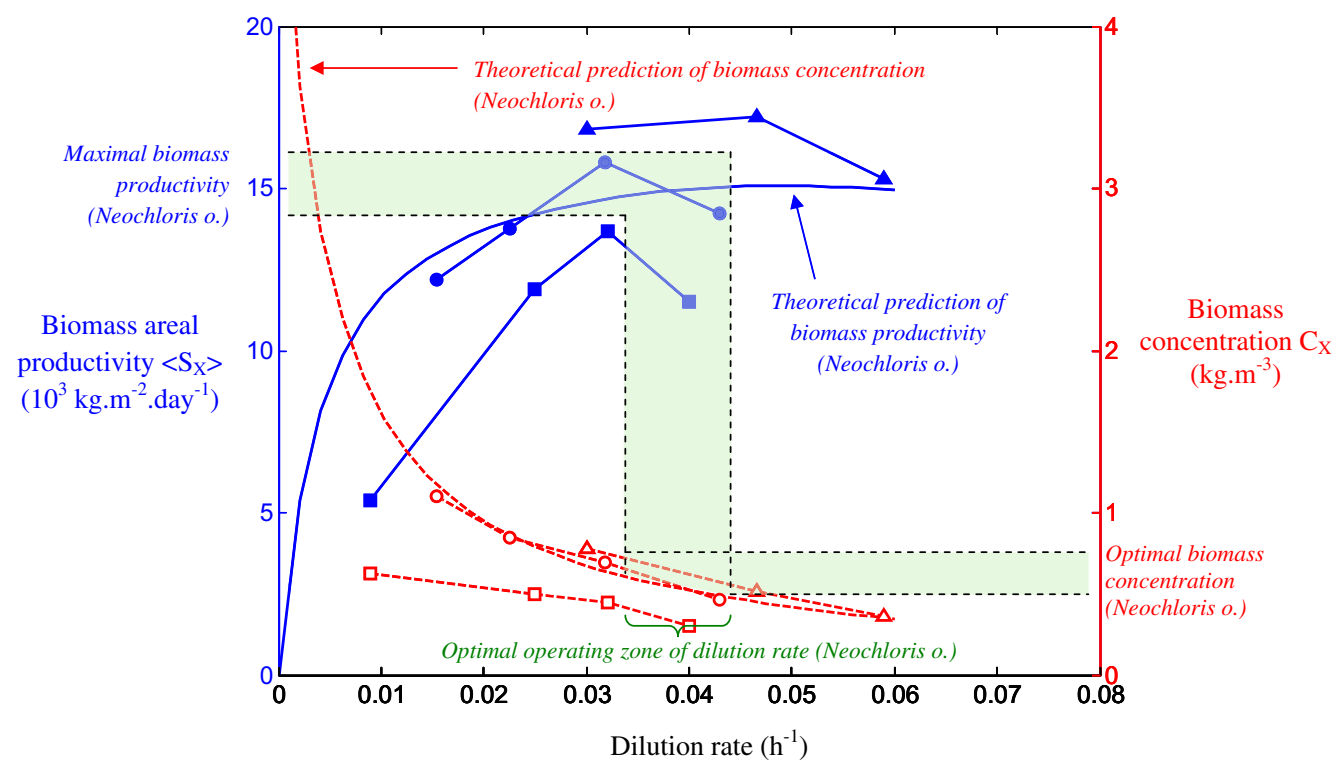

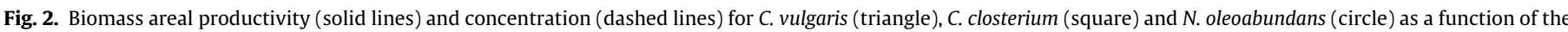

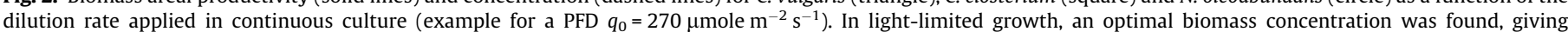

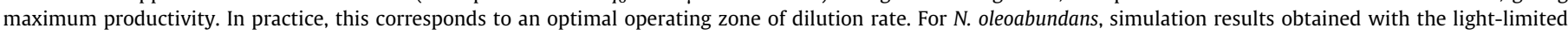
growth model are also given. 
on the kinetic performance of the process. There is thus an optimal biomass concentration, corresponding exactly to the appearance of the physical limitation by light (all light absorbed but no dark zone). This also corresponds to the maximum biomass productivity of the PBR (volume and surface).

The optimal biomass concentration can be sought experimentally (Takache et al., 2010) or theoretically (Cornet and Dussap, 2009). Experimental determination is straightforward and consists in varying the dilution rate with simultaneous measurement of steady-state biomass concentration $C_{x}$ to calculate corresponding volumetric biomass productivity $\left\langle r_{x}\right\rangle=D \cdot C_{x}$. Results are given in Fig. 2 for the three strains. Evolution of biomass productivity with the dilution rate was clearly observed, as well as the maximum biomass productivity. We note that almost the same maximum biomass productivity was achieved (Table 1), with $\left\langle S_{x}\right\rangle_{\max }=$ $0.016 \mathrm{~kg} \mathrm{~m}^{-2} \mathrm{day}^{-1} \quad\left(\left\langle r_{x}\right\rangle_{\max }=0.53 \mathrm{~kg} \mathrm{~m}^{-3} \mathrm{day}^{-1}\right), \quad\left\langle S_{x}\right\rangle_{\max }=0.017$ $\mathrm{kg} \mathrm{m}^{-2}$ day $^{-1} \quad\left(\left\langle r_{x}\right\rangle_{\max }=0.56 \mathrm{~kg} \mathrm{~m}^{-2} \mathrm{day}^{-1}\right)$ and $\left\langle S_{x}\right\rangle_{\max }=0.014$ $\mathrm{kg} \mathrm{m}^{-2}$ day $^{-1}\left(\left\langle r_{x}\right\rangle_{\max }=0.46 \mathrm{~kg} \mathrm{~m}^{-2}\right.$ day $\left.^{-1}\right)$ for $N$. oleoabundans, $C$. vulgaris and $C$. closterium respectively (all values accurate to $15 \%$ ). This confirms that differences among strains in light-limited growth conditions are only slight (at least for easy growing strains like those presented here). Analytical formulae (Eqs. (7) and (8)) enabling direct estimation of maximum productivity were tested. For $N$. oleoabundans a maximum areal biomass productivity $\left\langle S_{x}\right\rangle_{\max }=$ $0.015 \mathrm{~kg} \mathrm{~m}^{-2}$ day $^{-1}$ was obtained $\left(\left\langle r_{x}\right\rangle_{\max }=0.5 \mathrm{~kg} \mathrm{~m}^{-2}\right.$ day $\left.^{-1}\right)$. This clearly demonstrates here the interest and power of such a formula in the characterization of maximal performances of a given PBR and microalgal species (an acceptable deviation of $7 \%$ was obtained in this case).

Experimental investigation suffices to characterize the lightlimited growth and thus the maximum biomass productivity for given illumination conditions and PBR. However, the theoretical approach can be regarded as complementary as it allows a deeper characterization of the strain. Small-scale systems are sufficient to determine model parameters that can then be used to predict and optimize PBR productivity at higher scale, which is obviously of great relevance in the setting of mass scale systems for biodiesel fuel production. This is illustrated below in the scaling-up of biomass production for $N$. oleoabundans.

\subsection{Scaling-up of $N$. oleoabundans biomass production}

It is well known that PBR productivity is highly dependent on PBR geometry and operating conditions (especially light) (Takache et al., 2010). This explains the difficulty of comparing PBR technologies. Theoretical characterization, especially PBR modelling, is in this regard of prime interest, especially if the light-limited condition is applied. Biomass productivity can then be predicted using the same modelling approach as described above.

To illustrate this, $N$. oleoabundans was grown in an airlift PBR (PBR2) with higher volume $\left(V_{r}=0.13 \mathrm{~m}^{-3}\right)$ and greater depth of culture $\left(L_{z}=0.055 \mathrm{~m}\right)$, with illumination on two sides (total illuminated surface area $S=2.37 \mathrm{~m}^{2}$ ). Various PFD values were also applied, with a progressive increase to prevent photoinhibition

Table 1

Experimental and theoretical biomass productivity obtained in various conditions with $N$. oleoabundans.

\begin{tabular}{|c|c|c|c|}
\hline & Incident photons flux density $\left(\mu\right.$ mole $\left.\mathrm{m}^{-2} \mathrm{~s}^{-1}\right)$ & Experimental maximum biomass productivities & Theoretical maximum biomass productivities \\
\hline PBR1 $(L=3 \mathrm{~cm})$ & $q_{0}=270$ & $\begin{array}{l}\left\langle r_{x}\right\rangle_{\max }=0.53 \mathrm{~kg} \mathrm{~m}^{-3} \mathrm{day}^{-1} \\
\left\langle S_{x}\right\rangle_{\max }=16 \mathrm{~g} \mathrm{~m}^{-2} \mathrm{day}^{-1}\end{array}$ & $\begin{array}{l}\left\langle r_{x}\right\rangle_{\max }=0.5 \mathrm{~kg} \mathrm{~m}^{-3} \mathrm{day}^{-1} \\
\left\langle S_{x}\right\rangle_{\max }=15 \mathrm{~g} \mathrm{~m}^{-2} \mathrm{day}^{-1}\end{array}$ \\
\hline $\operatorname{PBR} 2(L=5.5 \mathrm{~cm})$ & $q_{0}=270$ & Not obtained (see text) & $\begin{array}{l}\left\langle r_{x}\right\rangle_{\max }=0.28 \mathrm{~kg} \mathrm{~m}^{-3} \mathrm{day}^{-1} \\
\left\langle S_{x}\right\rangle_{\max }=15.4 \mathrm{~g} \mathrm{~m}^{-2} \mathrm{day}^{-1}\end{array}$ \\
\hline $\operatorname{PBR} 2(L=5.5 \mathrm{~cm})$ & $q_{0}=360$ & Not obtained (see text) & $\begin{array}{l}\left\langle r_{x}\right\rangle_{\max }=0.33 \mathrm{~kg} \mathrm{~m}^{-3} \mathrm{day}^{-1} \\
\left\langle S_{x}\right\rangle_{\max }=18 \mathrm{~g} \mathrm{~m}^{-2} \mathrm{day}^{-1}\end{array}$ \\
\hline $\operatorname{PBR} 2(L=5.5 \mathrm{~cm})$ & $q_{0}=590$ & Not obtained (see text) & $\begin{array}{l}\left\langle r_{x}\right\rangle_{\max }=0.42 \mathrm{~kg} \mathrm{~m}^{-3} \mathrm{day}^{-1} \\
\left\langle S_{x}\right\rangle_{\max }=23 \mathrm{~g} \mathrm{~m}^{-2} \mathrm{day}^{-1}\end{array}$ \\
\hline
\end{tabular}

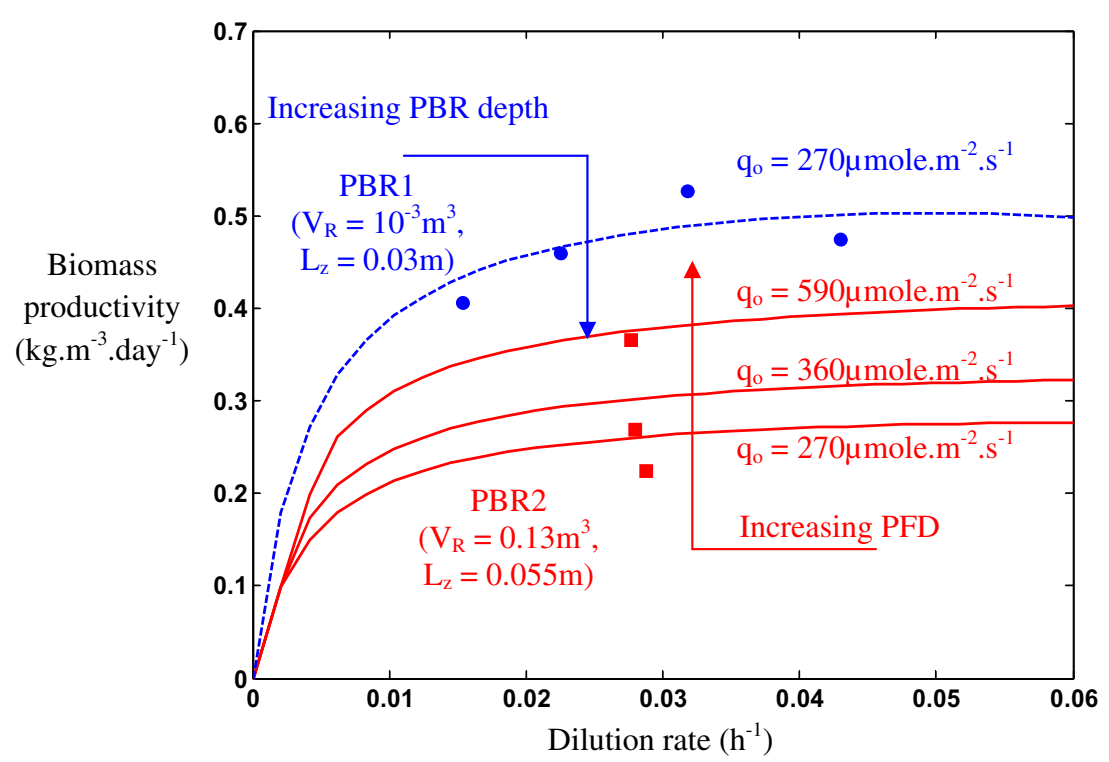

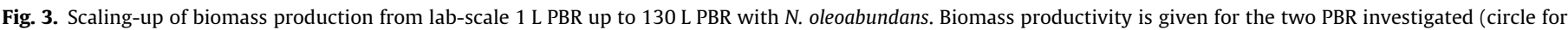

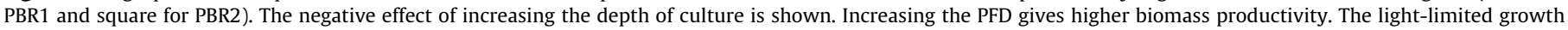
model can be used to predict the effect of different parameters on productivities or biomass concentration (dashed line for PBR1 and continuous line for PBR2). 
effects (progressive increase in biomass concentration and mutual shading). The results of both experimental and simulated productivities are given in Fig. 3. The usual increase in PBR productivity with increasing PFD values is observed. Decreasing PBR depth (and thus increasing $a_{\text {light }}$ ) also results in an increased biomass productivity. As often observed when increasing PBR size, we found lab-scale PBR to be more efficient (higher volumetric productivity) because of their reduced depth $\left(L_{z}=0.03 \mathrm{~m}\right)$. All evolutions (influence of PBR depth, PFD, dilution rate) were predicted by the lightlimited growth model, with a very close agreement between simulations and experiments. Maximum biomass productivity was also estimated using the general formula (Eq. (7)). Results are given in Table 1. For practical reasons, maximum productivity was not determined experimentally in PBR2 (as shown in Fig. 3, this would have implied a higher dilution rate, and so a large volume of medium). However, comparing theoretical results with values given in Fig. 3 shows that the theoretical calculation gives a good estimate of maximum productivity evolution for all incident PFDs. Because this is a direct analytical calculation (provided all parameters are defined), it offers a powerful tool to solve the problem of predicting productivities for different PBR geometries or operating conditions, as illustrated here with two PBRs with different volumes, illuminated surface areas and depths of culture, and run under different PFDs.

\subsection{Investigation of lipid production as a response to nitrogen starvation}

Nitrogen starvation induces several physiological changes in algae, deeply altering intracellular composition and growth kinetics. Biomass growth stops progressively, and lipid accumulation is triggered (concomitant with a marked decrease in pigment content). Lipid accumulation proved to be mainly TAGs, best suited to biodiesel production (Chisti, 2007; Hu et al., 2008; Pruvost et al.,
2009). Nitrogen starvation was thus applied here on all three strains. Because $C$. closterium was found to be less productive than other strains (in terms of maximum biomass productivity), only one experiment with partial analysis was conducted, and results are given here only for information. For the other two species, experiments were done in triplicate ( $N$. oleoabundans) and in duplicate $(C$. vulgaris). The results are given in Fig. 4 . As we see, a high reproducibility was achieved. This is explained here by the experimental procedure based on PBR cultivation in fully-controlled conditions. As described in Section 2.2, a PBR operating in continuous mode was used to produce algae in the same physiological state, and these cells were then inoculated in a second PBR where nitrate starvation was applied.

As reported by Pruvost et al. (2009), biomass growth was observed even though the cells were in nitrate-free medium. A major shift in cell composition was also observed, with a progressive lipid accumulation, a sudden increase in total sugar content from $20 \%$ to around $60 \%$ for $N$. oleoabundans ( $40 \%$ for C. vulgaris), and a significant protein content decrease from $60 \%$ to around $20 \%$ (for both species). This major reorganization is a direct consequence of nitrogen starvation, which triggers the accumulation of storage compounds such as sugar and lipids, with a concomitant degradation in nitrogen internal sources such as chlorophylls. Lipid contents are given in Table 2, in terms of total lipids and TAG contents (relative to dry weight). The effect of nitrogen starvation on TAG accumulation was clearly observed, whatever the strain. TAG content in standard growth conditions was very low $(<4 \%)$ but increased after four days of starvation up to $9-14 \%$ depending on the species. Although there was a difference in terms of total lipids (an increase was observed for $N$. oleoabundans and C. closterium, but a negligible variation was found for $C$. vulgaris), no great difference was observed among species in terms of TAG content. The highest content was in this case obtained with $C$. vulgaris (14\%). This last result emphasizes the need to distinguish between
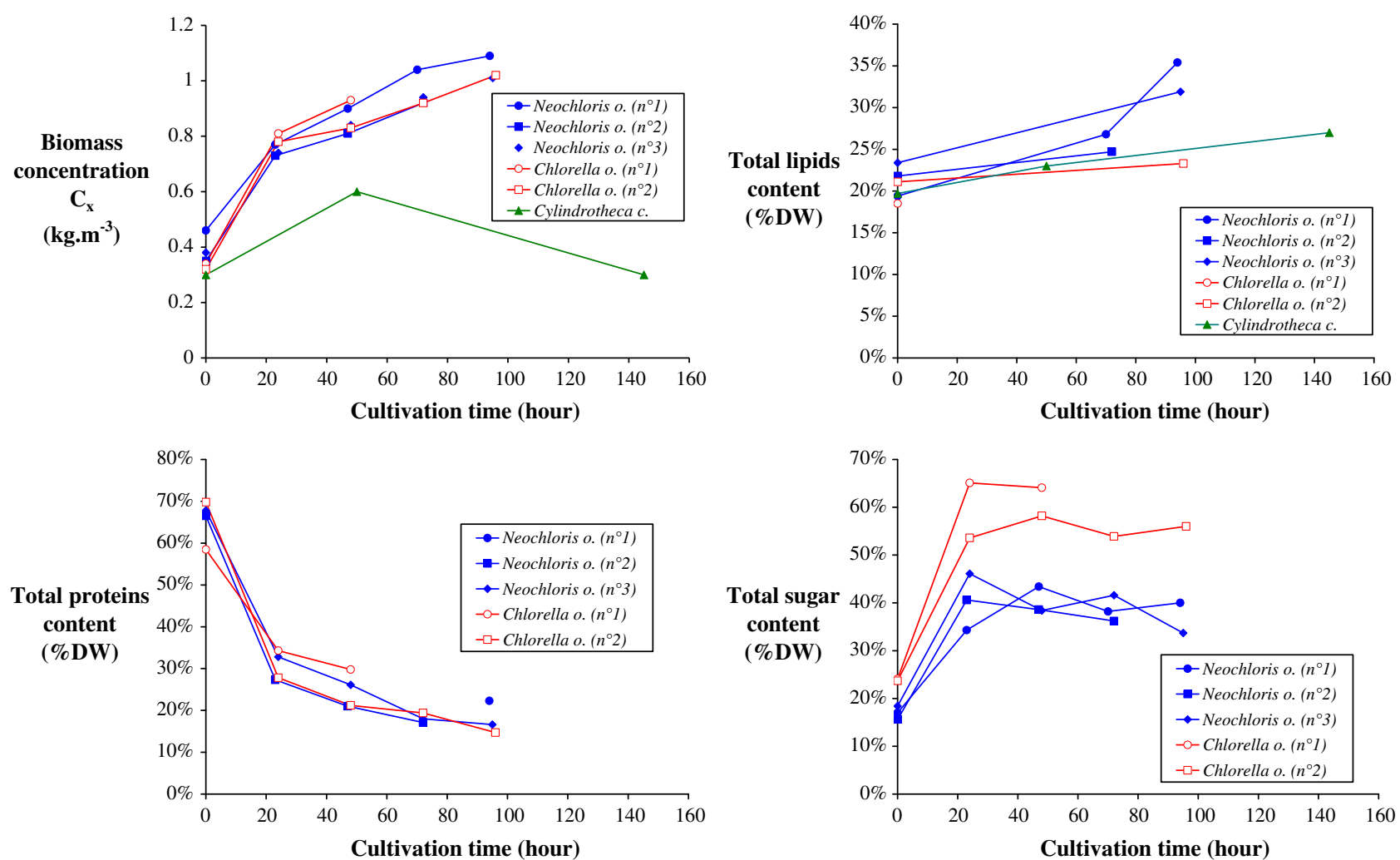

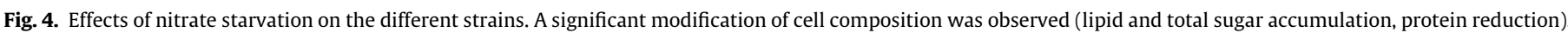


Table 2

Comparison of the three species for maximum productivities and lipid contents (total lipids and TAGs).

\begin{tabular}{|c|c|c|c|c|}
\hline & $\begin{array}{l}\text { Maximum biomass areal } \\
\text { productivities }\end{array}$ & $\begin{array}{l}\text { Maximum total (TL) and TAG areal } \\
\text { productivities }\end{array}$ & $\begin{array}{l}\text { Lipid content (optimal growth } \\
\text { conditions) }\end{array}$ & $\begin{array}{l}\text { Lipid content (nitrogen } \\
\text { starvation) }\end{array}$ \\
\hline $\begin{array}{l}\text { Neochloris } \\
\text { oleoabundans }\end{array}$ & $\begin{array}{l}\left\langle S_{x}\right\rangle_{\max }=16 \mathrm{~g} \mathrm{~m}^{-2} \text { day }^{-1} \\
\left(q_{0}=270 \mu \mathrm{mole} \mathrm{m}^{-2} \mathrm{~s}^{-1}\right)\end{array}$ & $\begin{array}{l}\left\langle S_{\mathrm{TL}}\right\rangle=3.5 \mathrm{~g} \mathrm{~m}^{-2} \mathrm{day}^{-1} \\
\left\langle S_{\mathrm{TAG}}\right\rangle=0.7-0.9 \mathrm{~g} \mathrm{~m}^{-2} \mathrm{day}^{-1} \\
\left(q_{0}=270 \mu \mathrm{mole} \mathrm{m}^{-2} \mathrm{~s}^{-1}\right)\end{array}$ & $\begin{array}{l}23 \% \text { of DW } \\
\text { (total lipids) } \\
<3 \% \text { of DW (TAG) }\end{array}$ & $\begin{array}{l}25-37 \% \text { of DW } \\
\text { (total lipids) } \\
9-12 \% \text { (TAG) }\end{array}$ \\
\hline Chlorella vulgaris & $\begin{array}{l}\left\langle S_{x}\right\rangle_{\max }=17 \mathrm{~g} \mathrm{~m}^{-2} \mathrm{day}^{-1} \\
\left(q_{0}=270 \mu \text { mole }^{-2} \mathrm{~s}^{-1}\right)\end{array}$ & $\begin{array}{l}\left\langle\mathrm{S}_{\mathrm{TL}}\right\rangle=3.5 \mathrm{~g} \mathrm{~m}^{-2} \mathrm{day}^{-1} \\
\left\langle\mathrm{~S}_{\mathrm{TAG}}\right\rangle=0.8-1.1 \mathrm{~g} \mathrm{~m}^{-2} \mathrm{day}^{-1} \\
\left(\mathrm{q}_{\mathrm{o}}=270 \mu \mathrm{mole}^{-2} \mathrm{~s}^{-1}\right)\end{array}$ & $\begin{array}{l}20 \% \text { of DW } \\
\text { (total lipids) } \\
<3 \% \text { of DW (TAG) }\end{array}$ & $\begin{array}{l}20-23 \% \text { of DW } \\
\text { (total lipids) } \\
11-14 \% \text { of DW (TAG) }\end{array}$ \\
\hline $\begin{array}{l}\text { Cylindrotheca } \\
\text { closterium }\end{array}$ & $\begin{array}{l}\left\langle S_{x}\right\rangle_{\max }=14 \mathrm{~g} \mathrm{~m}^{-2} \text { day }^{-1} \\
\left(q_{0}=270 \mu \text { mole }^{-2} \mathrm{~s}^{-1}\right)\end{array}$ & $\begin{array}{l}\left\langle\mathrm{S}_{\mathrm{TL}}\right\rangle=3 \mathrm{~g} \mathrm{~m}^{-2} \mathrm{day}^{-1} \\
\left\langle S_{\mathrm{TAG}}\right\rangle=0.2-0.3 \mathrm{~g} \mathrm{~m}^{-2} \mathrm{day}^{-1} \\
\left(q_{0}=270 \mu \mathrm{mole} \mathrm{m}^{-2} \mathrm{~s}^{-1}\right)\end{array}$ & $\begin{array}{l}17-20 \% \text { of DW } \\
\text { (total lipids) } \\
<3 \% \text { of DW (TAG) }\end{array}$ & $\begin{array}{l}20-30 \% \text { of DW } \\
\text { (total lipids) } \\
7-12 \% \text { of DW (TAG) }\end{array}$ \\
\hline
\end{tabular}

total lipids and TAG content (in terms of total lipids, N. oleoabundans showed the highest accumulation).

Table 2 also gives areal productivities for both total lipids and TAGs, using the same procedure as described in Pruvost et al. (2009). In continuous production, productivities were obtained from maximum biomass productivities and lipid natural content (normal growth conditions). In batch conditions, productivities were calculated as a function of cultivation time from biomass and lipid content time courses under nitrogen starvation. As in Pruvost et al. 2009, the highest productivity in terms of total lipids was found without mineral limitation (optimal growth conditions), with almost the same values whatever the strain (only the best results are given in Table 2). Because nitrogen starvation was necessary to trigger TAG accumulation, TAG productivities were deduced from batch experiments under nitrogen starvation. The best productivity was found with $C$. vulgaris (values in the range 0.8-1.1 $\mathrm{g} \mathrm{m}^{-2}$ day $^{-1}$ for the two batch cultivations), slightly higher than with $N$. oleoabundans (values in the range $0.7-0.9 \mathrm{~g} \mathrm{~m}^{-2}$ day $^{-1}$ for the three batch cultivations) as explained by higher TAG content for $C$. vulgaris. Lower productivity was obtained for $C$. closterium (0.2-0.3 $\left.\mathrm{g} \mathrm{m}^{-2} \mathrm{day}^{-1}\right)$. However, as stated above, only one cultivation was conducted for this last species.

\section{Conclusion}

A methodology combining experiments in lab-scale PBRs with a theoretical framework used to predict biomass productivity is presented for the systematic investigation of the potential of different microalgae species for biodiesel production. We emphasize the marked effects of nitrogen starvation, triggering TAG accumulation while affecting sugar and protein contents. A higher content of total lipids was achieved with $N$. oleoabundans (25-37\% of DW), while the highest TAG content was found in C. vulgaris (11-14\% of DW). These two species gave similar TAG productivities, with a slightly higher value for $C$. vulgaris $\left(1.1 \mathrm{~g} \mathrm{~m}^{-2} \mathrm{day}^{-1}\right)$ due to its higher TAG content.

\section{Acknowledgements}

This work was supported by the French National Research Agency as part of the "SHAMASH" ANR Program (French National Program), and by the CNRS Program LIP-ALG. The authors also thank Christine Fouque for her technical help.

\section{References}

Aiba, S., 1982. Growth kinetics of photosynthetic microorganisms. Advances in Biochemical Engineering and Biotechnology 23, 85-156.

Chisti, Y., 2007. Biodiesel form microalgae. Biotechnology Advances 25, 294-306.

Cogne, G., Lehmann, B., Dussap, C.-G., Gros, J.B., 2003. Uptake of macrominerals and trace elements by the cyanobacterium Spirulina platensis (Arthrospira platensis PCC 8005) under photoautotrophic conditions: culture medium optimization. Biotechnology and Bioengineering 81 (5), 588-593.
Cornet, J.-F., 2007. Procédés limités par le transfert de rayonnement en milieu hétérogène. Etude des couplages cinétiques et énergétiques dans les photobioréacteurs par une approche thermodynamique [Habilitation à Diriger des Recherches]. Université Blaise Pascal - Clermont-Ferrand, $\mathrm{n}^{\circ}$ d'ordre 236.

Cornet, J.F., Dussap, C.G., 2009. A simple and reliable formula for assessment of maximum volumetric productivities in photobioreactors. Biotechnology Progress 25, 424-435.

Cornet, J.F., Favier, L., Dussap, C.G., 2003. Modeling stability of photoheterotrophic continuous cultures in photobioreactors. Biotechnology Progress 19 (4), 12161227.

Degrenne, B., Pruvost, J., Christophe, G., Cornet, J.-F., Cogne, G., Legrand, J., 2010. Investigation of the combined effects of acetate and photobioreactor illuminated fraction in the induction of anoxia for hydrogen production by Chlamydomonas reinhardtii. International Journal of Hydrogen Energy, in press.

Doucha, J., Livansky, K., 2006. Productivity, $\mathrm{CO}_{2} / \mathrm{O}_{2}$ exchange and hydraulics in outdoor open high density microalgal (Chlorella sp.) photobioreactors operated in a Middle and Southern European climate. Journal of Applied Phycology 18, 811-826.

Dubois, M., Gilles, K.A., Hamilton, J.K., Rebers, P.A., Smith, F., 1956. Colorimetric method for determination of sugars and related substances. Analytical Chemistry 28, 350-356.

Fouchard, S., Pruvost, J., Degrenne, B., Legrand, J., 2008. Investigation of $\mathrm{H}_{2}$ production using the green microalga Chlamydomonas reinhardtii in a fully controlled photobioreactor fitted with on-line gas analysis. International Journal of Hydrogen Energy 33 (13), 3302-3310.

Fouchard, S., Pruvost, J., Degrenne, B., Titica, M., Legrand, J., 2009. Kinetic modeling of light limitation and sulphur deprivation effects in the induction of hydrogen production with Chlamydomonas reinhardtii part I: model description and parameters determination. Biotechnology and Bioengineering 102 (1), 132-147.

Gonzalez de la Vara, L., Gomez-Lojero, C., 1986. Participation of plastoquinone, cytochrome c553 and ferredoxin-NADP+ oxido reductase in both photosynthesis and respiration in Spirulina maxima. Photosynthesis Research $8,65-78$.

Hu, Q., Sommerfeld, M., Jarvis, E., Ghirardi, M.L., Posewitz, M., Seibert, M., Darzins, A., 2008. Microalgal triacylglycerols as feedstocks for biofuel production: perspectives and advances. The Plant Journal 54, 621-639.

Loubiere, K., Olivo, E., Bougaran, G., Pruvost, J., Robert, R., Legrand, J., 2009. A new photobioreactor for continuous microalgal production in hatcheries based on external-loop airlift and swirling flow. Biotechnology and Bioengineering 102 (1), 132-147.

Lowry, O.H., Rosebrough, N.J., Farr, A.L., Randall, R.J., 1951. Protein measurement with the Folin phenol reagent. Journal of Biological Chemistry 193, 265-275.

Mishchenko, M., Travis, L.D., 1998. Capabilities and limitations of a current FORTRAN implementation of the T-matrix method for randomly oriented, rotationally symmetric scatterers. Journal of Quantitative Spectroscopy and Radiative Transfer 60, 309-324.

Muller-Feuga, A., 1998. Growth as a function of rationing: a model applicable to fish and microalgae. Journal of Experimental Marine Biology and Ecology 236, 1-13.

Pottier, L., Pruvost, J., Deremetz, J., Cornet, J.F., Legrand, J., Dussap, C.G., 2005. A fully predictive model for one-dimensional light attenuation by Chlamydomonas reinhardtii in a torus reactor. Biotechnology and Bioengineering 91 (5), 569582.

Pruvost, J., Cornet, J.F., Legrand, J., 2008. Hydrodynamics influence on light conversion in photobioreactors: an energetically consistent analysis. Chemical Engineering Science 63, 3679-3694.

Pruvost, J., Van Vooren, G., Cogne, G., Legrand, J., 2009. Investigation of biomass and lipids production with Neochloris oleoabundans in photobioreactor. Bioresource Technology 100, 5988-5995.

Richmond, A., 2004. Handbook of Microalgal Culture: Biotechnology and Applied Phycology. Blackwell Sciences Ltd.

Ritchie, R.J., 2006. Consistent sets of spectrophotometric chlorophyll equations for acetone, methanol and ethanol solvents. Photosynthesis Research 89, 27-41.

Rodolfi, L., Chini Zittelli, G., Bassi, N., Padovani, G., Biondi, N., Bonini, G., Tredici, M. 2009. Microalgae for oil: strain selection, induction of lipid synthesis and outdoor mass cultivation in a low-cost photobioreactor. Biotechnology and Bioengineering 102 (1), 100-112.

Roels, J.A., 1983. Energetics and kinetics in biotechnology. Elsevier Biomedical Press, Amsterdam. 
Schreiber, U., Bilger, W., Hormann, H., Neubauer, C., 1998. Chlorophyll fluorescence as a diagnostic tool: basics and some aspects of practical relevance. In: Raghavendra, A.S. (Ed.), Photosynthesis: A Comprehensive Treatise. Cambridge University Press, Cambridge, pp. 320-336.

Scragg, A.H., Illman, A.M., Carden, A., Shales, S.W., 2002. Growth of microalgae with increased calorific values in a tubular bioreactor. Biomass and Bioenergy 23 (1), 67-73.

Strickland, J.D.H., Parsons, T.R., 1968. A practical handbook of seawater analysis: pigment analysis. Bulletin of Fisheries Research Board of Canada 167.
Takache, H., Christophe, G., Cornet, J.F., Pruvost, J., 2010. Experimental and theoretical assessment of maximum productivities for the micro-algae Chlamydomonas reinhardtii in two different geometries of photobioreactors. Biotechnology Progress 26 (2), 431-440.

Tornabene, T.G., Holzer, G., Lien, S., Burris, N., 1983. Lipid composition of the nitrogen starved green alga Neochloris oleoabundans. Enzyme Microbial Technology 5, 435-440.

Van de Hulst, H., 1981. Light Scattering by Small Particle, second ed. Dover Publications Inc., New York. 